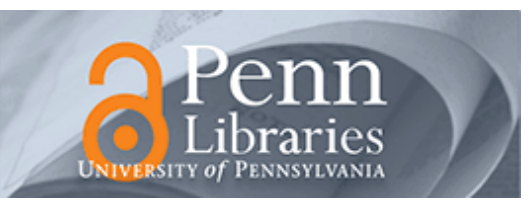

University of Pennsylvania

ScholarlyCommons

$10-2007$

\title{
Psychiatric Comorbidity and Greater Hospitalization Risk, Longer Length of Stay, and Higher Hospitalization Costs in Older Adults with Heart Failure
}

\author{
Steven L. Sayers \\ University of Pennsylvania \\ Nancy Hanrahan \\ Ann Kutney Lee \\ University of Pennsylvania \\ Sean P. Clarke \\ Brendali F. Reis
}

See next page for additional authors

Follow this and additional works at: https://repository.upenn.edu/nrs

Part of the Cardiology Commons, Cardiovascular Diseases Commons, Circulatory and Respiratory

Physiology Commons, Geriatrics Commons, Health and Medical Administration Commons, Medical

Humanities Commons, Nursing Commons, Psychiatry Commons, and the Psychiatry and Psychology

Commons

\section{Recommended Citation}

Sayers, S. L., Hanrahan, N., Kutney Lee, A., Clarke, S. P., Reis, B. F., \& Riegel, B. (2007). Psychiatric Comorbidity and Greater Hospitalization Risk, Longer Length of Stay, and Higher Hospitalization Costs in Older Adults with Heart Failure. Journal of the American Geriatrics Society, 55 (10), 1585-1591. http://dx.doi.org/10.1111/j.1532-5415.2007.01368.x 


\title{
Psychiatric Comorbidity and Greater Hospitalization Risk, Longer Length of Stay, and Higher Hospitalization Costs in Older Adults with Heart Failure
}

\begin{abstract}
OBJECTIVES: To explore associations between psychiatric comorbidity and rehospitalization risk, length of hospitalization, and costs.

DESIGN: Cross-sectional study of 1-year hospital administrative data.

SETTING: Claims-based study of older adults hospitalized in the United States.

PARTICIPANTS: Twenty-one thousand four hundred twenty-nine patients from a $5 \%$ national random sample of U.S. Medicare beneficiaries aged 65 and older, with at least one acute care hospitalization in 1999 with a Diagnostic-Related Group of congestive heart failure.
\end{abstract}

MEASUREMENTS: The number of hospitalizations, mean length of hospital stay, and total hospitalization costs in calendar year 1999.

RESULTS: Overall, $15.8 \%$ of patients hospitalized for heart failure (HF) had a coded psychiatric comorbidity; the most commonly coded comorbid psychiatric disorder was depression $(8.5 \%$ of the sample). Most forms of psychiatric comorbidity were associated with greater inpatient utilization, including risk of additional hospitalizations, days of stay, and hospitalization charges. Additional hospitalization costs associated with psychiatric comorbidity ranged up to $\$ 7,763$, and additional days length of stay ranged up to 1.4 days.

CONCLUSION: Psychiatric comorbidity appears in a significant minority of patients hospitalized for HF and may affect their clinical and economic outcomes. The associations between psychiatric comorbidity and use of inpatient care are likely to be an underestimate, because psychiatric illness is known to be underdetected in older adults and in hospitalized medical patients.

\section{Keywords}

heart failure, psychiatric comorbidity, depression, hospitalization costs, economic analysis, Medicare, sex, socioeconomic status, race, ethnicity

\section{Disciplines}

Cardiology | Cardiovascular Diseases | Circulatory and Respiratory Physiology | Geriatrics | Health and Medical Administration | Medical Humanities | Medicine and Health Sciences | Nursing | Psychiatry | Psychiatry and Psychology

\section{Author(s)}

Steven L. Sayers, Nancy Hanrahan, Ann Kutney Lee, Sean P. Clarke, Brendali F. Reis, and Barbara Riegel 
Psychiatric Comorbidity and Increased Hospitalization Risk, Length of Stay, and Hospitalization Costs in Older Adults with Heart Failure

Steven L. Sayers, PhD, *† Nancy Hanrahan, PhD RN, $\ddagger$ Ann Kutney, RN MSN, $\ddagger$

Sean P. Clarke, PhD, RN, , FAAN $\ddagger$ Brendali F. Reis, Ph.D.,§ and Barbara Riegel, DNSc, RN, FAAN\|

*VISN 4 Mental Illness, Research, Education and Clinical Center, Philadelphia Veterans Affairs Medical

Center, †Department of Psychiatry of the University of Pennsylvania, ¥Center for Health Outcomes and Policy Research, School of Nursing, University of Pennsylvania, §Institute for Graduate Clinical

Psychology, Widener University, \| School of Nursing and Leonard Davis Institute,

University of Pennsylvania

Word count of text: 2988

Running head: Heart Failure and Psychiatric Comorbidity

Corresponding author:

Steven L. Sayers, Ph.D.

Philadelphia VA Medical Center

MIRECC 116

3900 Woodland Avenue

Philadelphia, PA 19104

(215) 823-5196

steven.sayers@va.gov
Alternate corresponding author: Nancy Hanrahan, PhD RN

nancyp@nursing.upenn.edu

Preparation of the current report was supported by grants from the American Heart Association. We also appreciate the support of the VISN 4 Mental Illness Research, Education, and Clinical Center (MIRECC), Veterans Affairs Medical Center, Philadelphia, Pennsylvania. Portions of the current findings were presented at the $9^{\text {th }}$ Annual Meeting of the Heart Failure Society of America, September, 2005, Boca Raton, FL. We acknowledge the support from the Center for Health Outcomes and Policy Research, School of Nursing, University of Pennsylvania, under the National Institute for Nursing Research grants “Center for Nursing Outcomes Research” (P30-NR-005043, Linda H. Aiken, PI) and “Advanced Training in Nursing Outcomes Research” (T32-NR-007104, Linda H. Aiken, PI). 
1

2 OBJECTIVES: To explore associations of psychiatric comorbidity with rehospitalization risk,

3 length of hospitalization, and costs.

4 DESIGN: Cross-sectional study of 1-year hospital administrative data.

5 SETTING: Claims-based study of older adults hospitalized in the United States.

6 PARTICIPANTS: 21,429 patients from a 5\% national random sample of U.S. Medicare

7 beneficiaries, aged 65 and older, with at least one acute care hospitalization in 1999 with a

8 Diagnostic-Related Group of Congestive Heart Failure.

9 MEASUREMENTS: The number of hospitalizations, mean length of hospital stay, and total

10 hospitalization costs in calendar year 1999.

11 RESULTS: Overall, 15.82\% of patients hospitalized for heart failure had a coded psychiatric comorbidity; the most commonly coded comorbid psychiatric disorder was depression (8.5\% of the sample). Most forms of psychiatric comorbidity were associated with increases in inpatient utilization, including risk of additional hospitalizations, additional days of stay, and additional hospitalization charges. Additional hospitalization costs associated with psychiatric comorbidity ranged up to $\$ 7763$ and additional days length of stay ranged up to 1.4 days.

CONCLUSION: Psychiatric comorbidity appears in a significant minority of patients hospitalized for heart failure and may affect their clinical and economic outcomes. The associations of psychiatric comorbidity with utilization of inpatient care are likely to be an underestimate because psychiatric illness is known to be underdetected in older adults and in hospitalized medical patients. 
22 KEYWORDS: Heart failure, psychiatric comorbidity, depression, hospitalization costs, economic 23 analysis, Medicare, gender, socioeconomic status, race/ethnicity

24 Word count of abstract: 214 words 


\section{INTRODUCTION}

Currently, over 5 million Americans have heart failure, ${ }^{1}$ with increasing prevalence in older cohorts of adults, largely due to the aging U.S. population and increased survival of patients with heart failure. Despite medical advances ${ }^{2}$ and the positive impact of heart failure management programs, ${ }^{3}$ the cost of care for heart failure remains high. Heart failure is responsible for $\$ 33.2$ billion in health care costs in the U.S., approximately $1.5 \%$ of total health care expenditure. ${ }^{3}$ Hospitalizations account for $65 \%$ of the costs of treating heart failure. ${ }^{3}$

There is significant evidence that comorbid psychiatric disorders contribute to increased risk of hospitalization among patients with heart failure and increased costs of these hospitalizations. ${ }^{4}$ Depression is common among hospitalized older adults with heart failure,${ }^{5}$ and likely leads to increased outpatient utilization, higher health care costs, and to a doubled risk of rehospitalization and death within 1 year. ${ }^{6}$ Few studies have examined the consequences of a broad range of psychiatric disorders for hospitalized older adults with heart failure (e.g., anxiety disorders, alcohol abuse), despite evidence of increased rates of these problems in the heart failure population. $^{7}$

The goal of this study was to examine the effects of identified comorbid psychiatric disorders on healthcare utilization and costs in a sample of Medicare beneficiaries hospitalized at least once with a primary diagnosis of heart failure in 1999. Our major hypothesis was that having a psychiatric disorder would be associated with increased rates of hospitalization, length of hospitalizations, and hospitalization costs in older adults with heart failure. Consistent with existing findings we hypothesized that identified comorbid psychiatric disorders might vary across groups defined by gender, socioeconomic status, ${ }^{8}$ and race $^{9}$ and that these variables, as well as 
47 medical comorbidities, would require controls in our models examining the association between 48 psychiatric comorbidities and health care utilization. 


\section{METHODS}

\section{Study Sample}

52 The study sample was drawn from a 5\% random national sample of Medicare beneficiaries

53 hospitalized during the 1999 calendar year. Using the Denominator and MedPAR files obtained

54 from the Center for Medicaid and Medicare Services (CMS), we extracted records of

55 hospitalizations in 1999 that were assigned the Congestive Heart Failure Diagnostic-Related

56 Grouping (DRG 127). Records of different hospitalizations for the same patients were then

57 collapsed so that the patient became the unit of analysis for most analyses. Because we used

58 hospital DRGs to identify target patients, we excluded patients for whom heart failure was not a

59 primary cause of any admission during 1999, even though it might have been a contributory cause

60 for an admission during that year. This approach was used to limit the extent to which we included

61 patients without a chronic heart failure condition. We excluded patients with comorbid dementia or

62 organic brain syndrome diagnosis from the sample because they might exhibit less variability in

63 their clinical course and the associated costs compared to those with other psychiatric

64 disturbances. ${ }^{10,11}$

Of the $5 \%$ national sample of 357,232 unique patients, a total of 27,949 patients had a CHF

66 DRG during at least one of their hospitalizations for that year. About one-fifth of these patients

67 were excluded due to dementia or organic brain syndrome $(n=3,123,11.1 \%)$, or an age less than 65

68 years $(n=2,628,9.4 \%)$ because persons younger than 65 years may have qualified for Medicare

69 coverage on the basis of physical or mental disability. A further $2.8 \%(n=769)$ were excluded on

70 the basis of having been admitted before the year 1999, hospitalization costs suggesting data entry

71 errors, or stays longer than 365 days suggestive of long-term care rather than acute 
72 hospitalizations. The final sample included 21,429 individuals with a CHF DRG for at least one of

73 their hospital stays during the year 1999.

\section{$74 \quad$ Measures}

75

76

77

78

79

80

81

82

84

85

86

87

88

89

90

91

92

We used the definitions of medical comorbidity indices as described by Elixhauser and colleagues $^{12}$ and used their algorithm as applied in publicly-available software code distributed by the Agency for Healthcare Research and Quality (AHRQ). ${ }^{13}$ The algorithm places emphasis on comorbidities that could increase resources used or the risk of poor outcomes that are not directly related to the reason for admission. ${ }^{12}$ Because of our focus on heart failure, it was not analyzed as a separate comorbidity in our analyses.

For the current study we expanded Elixhauser and colleagues' ${ }^{32}$ categories of comorbid psychiatric disorders based on the initial work by Hanrahan, ${ }^{14}$ as well as evidence for inclusion of disorders not represented in the system. ${ }^{7}$ Psychiatric diagnoses within each category were identified using AHRQ’s Clinical Classifications Software ${ }^{15}$ (available from the first author upon request). Our final list of psychiatric comorbidity categories was as follows: alcohol abuse, drug abuse, psychosis, depression, bipolar disorders, anxiety disorders, and other psychiatric conditions. The variable we analyzed was presence vs. absence in any hospitalizations in 1999 of each type of comorbid medical and psychiatric condition.

The following demographic variables were analyzed: age, gender, and race. In addition, we used the receipt of Medicaid benefits anytime during the year 1999 as a proxy for socioeconomic status because so-called “dual” eligibility for Medicaid and Medicare requires falling below poverty levels defined by the U.S. Department of Health and Human Services. ${ }^{16}$ 
Our primary health care utilization and outcome included the patient's total number of

94 hospitalizations in 1999, average length of stay (LOS) of all hospitalizations in 1999, and total

95 hospitalization charges in 1999. All indices were collapsed across patients' hospitalizations for the 96 year and represented the values for individual patients.

\section{Statistical Analyses}

Rates of psychiatric comorbidity were calculated, along with 95\% confidence intervals.

Relative proportions of the sample of patients with psychiatric diagnosis by gender, race, and lowincome status were examined using 2 X 2 chi-square tests. The continuous outcomes of number of

101 hospitalizations, average LOS, and total charges were all markedly positively skewed, but after logarithmic data transformation they approximated the normal distribution. These transformations were reversed for presentation purposes in the tables. The data were evaluated for amount,

104 distribution, and patterns of missing data, out-of-range values, plausible means, and univariate outliers. Missing values were randomly scattered throughout and were less than $5 \%$ of the data points. Cases with incomplete data on the variables relevant for each analysis were dropped. Group differences were examined using $t$-tests and Satterthwaite's ${ }^{17}$ method for estimating degrees of

108 freedom for unequal variances where appropriate. using regression modeling where the patient was the unit of analysis. Generalized linear modeling was used to test the hypothesis that psychiatric comorbidity explained a greater number of

112 hospitalizations, mean LOS, and total hospitalization charges. Each of these analyses included the

113 binomial variables (presence/absence) of each type of medical comorbidity as defined by

114 Elixhauser et al., ${ }^{12}$ entered as a block along with the expanded psychiatric comorbidity

115 categories. Because the continuous outcomes of hospitalizations, average LOS, and total charges 
116 were all positively skewed, we considered log transformation, but we were concerned about

117 retransformation bias. In order to select the correct model, we examined the distributions of the

118 log-scale residuals from GLM models with a log link function following the method of Manning

119 and Mullahy. ${ }^{18}$ There was little kurtosis on the log-scale residuals (i.e., kurtosis $<3$ ) so we chose

120 GLM over ordinary least squares regression. Modified Park tests on the raw-scale residuals

121 indicated that $\lambda=2$ for each outcome, so the gamma family was selected for the final GLM models.

122 To illustrate the impact of comorbidities on the outcome variables, estimates of effects of all

123 comorbidity parameters from these models were transformed from the log values used for the

124 analysis and then presented in a table. For parameters that were statistically significant, entries

125 tabled were calculated using the parameter estimate for each effect and the intercept values of the

126 major outcomes of interest that resulted from the significant model.

127 
128

129

130

131

132

133

134

\section{RESULTS}

\section{Sample characteristics}

Table 1 presents the demographic and clinical characteristics of the final sample of 21,429 heart failure patients. Over half of the patients were female (58.8\%), and most were white (84.9\%), and all were at least 65 years of age. Approximately one-fifth of the sample was designated as lowincome and 33.8\% died during the 1999 year. As shown in Table 1, the mean total hospitalization charges was $\$ 40,048$ but this outcome was highly skewed-the median value was $\$ 22,934$.

\section{Prevalence of psychiatric comorbidities in the sample}

Table 2 presents prevalence estimates of identified psychiatric comorbidity, drawn from every hospitalization of each patient in the sample. A significant minority of patients had at least one psychiatric comorbid condition recorded. Within this subset of patients, $15.8 \%(\mathrm{~N}=518)$ of patients had 2 or more different psychiatric diagnoses within or across any of their hospitalizations in that year. However, these patients did not have systematically greater patterns of hospitalization utilization. Those with more than one psychiatric diagnosis, compared to those with only one, had a greater number of hospitalizations, $M=5.5, S D=3.4$, vs. $M=4.0, S D=2.6, t(639)=9.86$, $\mathrm{p}<$ .0001 , but lower average costs per hospitalization, $M=\$ 11,845, S D=\$ 8,566$, vs. $M=\$ 12,914$, $S D=\$ 10,766, t(835)=-2.51, p<.05$. The two groups did not differ on the mean length of hospitalizations, $M=8.8, S D=6.1$, vs. $M=8.6, S D=7.5, t(835)=0.58, p>.05$.

\section{Psychiatric comorbidities in relation to gender, socioeconomic status, and race}

Women were more likely than men to have a comorbid psychiatric diagnosis, $16.8 \%$ vs. $13.3 \%, \chi^{2}[1]=48.82, p<.0001, \mathrm{~N}=21,429$. Depression and anxiety disorders were more common in women $\left(10.0 \%\right.$ vs. $6.3 \%, \chi^{2}[1]=89.77$, and $5.0 \%$ vs. $2.1 \%, \chi^{2}[1]=114.01$, 
150 respectively, both $p$ 's $<.0001$ ). Male patients were more likely than female patients to have an

151 alcohol abuse diagnosis ( $2.5 \%$ vs. $\left.0.5 \%, \chi^{2}[1]=157.57, p<.0001\right)$. No differences in rates of

152 bipolar disorder, psychoses, drug abuse, or other psychiatric problems were observed by gender.

153 A higher proportion of low-income beneficiaries had psychiatric diagnoses, compared to all

154 other beneficiaries, $18.7 \%$ vs. $14.4 \%, \chi^{2}[1]=50.74, p<.0001, \mathrm{~N}=21,429$. Specifically, more

155 low-income patients had diagnoses of psychosis ( $3.4 \%$ vs. $\left.2.2 \%, \chi^{2}[1]=21.38, p<.0001\right)$,

156 depression $\left(10.1 \%\right.$ vs. $\left.8.1 \%, \chi^{2}[1]=18.33, p<.0001\right)$, drug abuse $\left(0.9 \%\right.$ vs. $0.5 \%, \chi^{2}[1]=10.36, p$

$157<.005)$, anxiety disorders ( $4.7 \%$ vs. $\left.3.6 \%, \chi^{2}[1]=14.17, p<.001\right)$, and other psychiatric diagnoses

$158\left(1.8 \%\right.$ vs. $\left.1.0 \%, \chi^{2}[1]=21.24, p<.0001\right)$. There were no differences in the likelihood of bipolar

159 disorder or alcohol abuse disorders by income status.

160 Proportionally more White patients had a recorded psychiatric diagnosis than non-White

161 patients (Black and other non-White categories combined), $15.8 \%$ vs. $12.4 \%, \chi^{2}[1]=23.25, p<$

162 .0001). Fewer Black patients had psychiatric diagnoses than those in the White or other race

163 categories combined, $12.5 \%$ vs. $15.7 \%, \chi^{2}[1]=16.3, p<.0001$.

164 Psychiatric comorbidities as predictors of outcomes

165 Number of hospitalizations. Consistent with our hypotheses, all types of psychiatric

166 comorbidity were associated with a greater risk of hospitalizations, $\chi^{2}(38)=4008.30, p<.0001$,

167 after adjusting for medical comorbidities (see Table 3). All forms of psychiatric comorbidity were

168 associated with having at additional risk of hospitalization, ranging from .20 to .39 additional

169 hospitalizations, on average. Sensitivity analyses excluding patients who had died during 1999

170 revealed very similar findings indicating no significant effects due to an increase in number of

171 hospitalizations at end of life. 
Follow-up analyses indicated psychiatric comorbidity was associated with increased risk of

173 hospitalizations specifically attributed to heart failure exacerbation (i.e., with a DRG of heart

174 failure). The mean heart failure hospitalizations for patients with psychiatric comorbidity versus no

175 psychiatric comorbidity, were $M=1.59(S D=1.11), M=1.35(S D=.80)$ and respectively, $t(3921)=$ -

$176 \quad 12.16, p<.0001$.

Mean length of stay (LOS). In partial support of our hypothesis, alcohol abuse, psychoses, major depression, and bipolar disorder were significant predictors of increased mean LOS, after

179 adjusting for the effects of all significant types of medical comorbidity, $\chi^{2}(38)=1383.76, p<$

180 .0001. Significantly, having bipolar disorder as a comorbid diagnosis was associated with 1.4

181 additional days of stay per hospitalization on average, holding other factors constant. The effects

182 of each type of psychiatric comorbidity were independent of each other and were similar in

183 magnitude when tested individually, indicating no significant multicollinearity effects. Sensitivity

184 analyses showed no impact of excluding patients who died during the study year, suggesting that

185 the findings could not be attributed to an "end-of-life" effect.

Total hospital charges. Consistent with our hypotheses, psychiatric comorbidities, as a

187 group, were associated with higher total hospitalization costs, $\chi^{2}(38)=7294.32, p<.0001$. As

shown in Table 3, the additional hospitalization costs specifically attributable to each diagnosis,

189 ranged from $\$ 1,8433.89$ - $\$ 7,763.47$. Again, parallel analyses indicated that the effects of

190

psychiatric comorbidity were not dependent on the multicollinearity of the variables. Sensitivity

191 analyses indicated no effect associated with hospital charges by patients at the end of life. 


\section{DISCUSSION}

194

195

196

197

198

199

200

201

202

203

204

205

206

207

These findings represent the first comprehensive examination of psychiatric comorbidity and hospitalizations of older patients with heart failure. We have presented evidence that the negative effects of depression on the course of heart failure ${ }^{19}$ may extend to other psychiatric disturbances. In a nationally representative sample we showed that psychiatric comorbidity was prevalent in Medicare recipients hospitalized for heart failure, with depression being the most common form. All forms of psychiatric comorbidity were associated with risk of additional hospitalizations comparable to medical comorbidities within a 1-year period and that the presence of alcohol abuse, depression, bipolar disorder, or psychoses predicted longer hospitalizations. All forms of psychiatric comorbidity were associated with increased health care costs that were at least comparable to other major forms of chronic illness. For example, diabetes with chronic complications was associated with 30\% increased costs; depression and bipolar disorder were associated with cost increases of 25\% and 52\%, respectively. Analyses reported in this paper accounted for the effects of medical comorbidities using a method developed specifically to adjust for their impact on health care utilization, costs, and illness burden. ${ }^{12}$

There are several potential explanations for these effects. Heavy cardiac and noncardiac comorbidities in older adults with heart failure complicates their treatment. ${ }^{20}$ It may be more difficult to stabilize patients who are depressed or psychotic, and the functional disability associated with these conditions is a barrier to effective treatment. ${ }^{21}$ Those with psychiatric disturbances have poorer treatment adherence and self-care. ${ }^{22}$ There is also evidence that even when psychiatric disturbances are recognized and initially treated in medical contexts, the psychiatric outcomes of these patients are no better than those who had psychiatric disturbance 
215 unrecognized by their medical providers. ${ }^{23,}{ }^{24}$ Thus, research and clinical innovation will be

216 needed to address the challenge of treating psychiatric problems in medical settings.

217 In addition, activation of the hypothalamic-pituitary-adrenocortical axis associated with

218 psychiatric disturbance may play a role in poorer course within and between hospitalizations,

219 although this type of explanation has most typically been applied to the role of depression in

220 cardiovascular outcomes. ${ }^{25}$ Our data do point to effects of psychiatric comorbidity on the course of

221 HF, specifically; patients with psychiatric comorbidity had a greater number of HF

222 hospitalizations. Although our data cannot specifically address the mechanisms involved, this is an

223 important avenue for future research.

$224 \quad$ Although out findings are similar to those of Braunstein and colleagues ${ }^{6}$ we used more 225 restrictive criteria to identify patients with chronic heart failure (i.e., at least one hospitalization 226 with a Congestive Heart Failure DRG). This likely resulted in a more severely ill patient sample.

227 This restriction may explain why $35.3 \%$ of our sample died during the year on which the sample is 228 based, compared to $18 \%$ of the sample defined by Braunstein. Our findings extend earlier work by 229 showing that other psychiatric disorders in addition to depression have important associations with 230 increased medical care utilization and costs.

231 There were limitations resulting from the use of claims-based administrative data. There 232 might have been positive and/or negative biases in diagnosis and medical record coders that 233 resulted from sources such as payment incentives and stigma associated with psychiatric 234 diagnoses. ${ }^{26}$ The diagnoses in the current study could not be validated against standardized criteria. 235 Although other studies have reported adequate sensitivity and specificity for chart based 236 psychiatric diagnoses in mental health settings, ${ }^{27}$ it is likely that diagnoses obtained from claims237 based records in the current study led to lower sensitivity for psychiatric disorders. Mood 
238 disorders, and potentially other psychiatric problems, are significantly underdiagnosed and

239 undertreated in older adults in medical settings. ${ }^{24}$ The rates of depression we reported are lower

240 than in interview studies of carefully diagnosed patients hospitalized for heart failure (i.e., ranging

241 from 20 - 37\%). ${ }^{5}$ In addition, the phenomenon of treatment of psychiatric disorders without

242 chart documentation of a psychiatric disorder ${ }^{29}$ indicate that psychiatric difficulties in our sample

243 could be higher than the chart-based rates suggest.

244 No information was available regarding the severity of heart failure in the sample.

245 However, our method of replicating analyses without patients who later died gave some control

246 over illness severity. We cannot exclude the possibility that outcomes may be worse for patients

247 with coded comorbid psychiatric diagnoses as opposed to the presence of the conditions

248 themselves. In addition, only longitudinal designs, with more carefully diagnosed psychiatric

249 conditions, can clarify the complex causal relationships between psychiatric conditions and the

250 course of heart failure hospitalizations and outcomes.

\section{CONCLUSION}

Psychiatric comorbidity is prevalent among older adults with heart failure and our findings suggest that psychiatric illness has a demonstrable impact on the utilization of acute inpatient medical care. Future research should focus on the how this effect occurs. Important changes in clinical practice with these patients may include routine screening for psychiatric disorder and increased access to pharmacological as well as cognitive-behavioral interventions, with modifications based on the needs of older adults. Future research also should be focused on how best to identify and treat psychiatric disturbance in older patients with heart failure. This line of research has promise in reducing the burden of illness on older adults with heart failure, and in 260 reducing the high costs of treating patients with this condition. 


\section{ACKNOWLEDGMENT}

262 Financial Disclosures:

263 Steven L. Sayers: Dr. Sayers appreciates the support of grants from the American Heart

264 Association, as well as in-kind support from the VISN 4 Mental Illness Research, Education, and

265 Clinical Center (MIRECC), Veterans Affairs Medical Center, Philadelphia, Pennsylvania.

266 Nancy Hanrahan, Ann Kutney, Sean Clarke: Drs. Hanrahan and Clarke, and Ms. Kutney

267 appreciate the support from the Center for Health Outcomes and Policy Research, University of

268 Pennsylvania School of Nursing and National Institute for Nursing Research grants "Center for

269 Nursing Outcomes Research” (P30-NR-005043, Linda H. Aiken, PI) and “Advanced Training in

270 Nursing Outcomes Research” (T32-NR-007104, Linda H. Aiken, PI).

271 Brendalis Reis: None

272 Barbara Riegel: Dr. Riegel acknowledges the support of grants from the National Institutes of

273 Health and the Kynett Foundation.

274 Author Contributions:

275 Steven L. Sayers, Nancy Hanrahan, Ann Kutney, Sean Clarke, Brendalis Reis, and Barbara

276 Riegel: These authors were involved in the concept and design of the study, as well as the

277 interpretation of data analyses and preparation of the manuscript.

278 Steven L. Sayers, Nancy Hanrahan, Ann Kutney, and Sean Clarke: These authors were directly

279 responsible for obtaining the data and performing the data analyses.

280 Sponsor's Role: Not applicable.

281 The Centers for Medicare \& Medicaid Services (CMS) have reviewed the subject

282 manuscript and approve its use as submitted. This approval is based on the use of CMS data and

283 does not represent a CMS endorsement of, or comment on, the actual manuscript itself. 
284 Timothy P. Cheney, Center for Health Outcomes and Policy Research, and John E. Marcotte, 285 Ph.D., Center for Population Studies, University of Pennsylvania, contributed statistical and programming 286 expertise to the study. Thanks are extended to Ira R. Katz, M.D., Ph.D., of the University of 287 Pennsylvania and the VISN 4 MIRECC, Joseph Gallo, M.D., Ph.D., of the University of 288 Pennsylvania, and Linda H. Aiken, Ph.D., FAAN, FRCN, RN, Center for Health Outcomes and 289 Policy Research, University of Pennsylvania School of Nursing, for their comments and 290 suggestions on a previous version of the manuscript. 
Table 1. Demographic and Clinical Characteristics of Patients in the 5\% Sample With a CHF DRG At Any Hospitalization During 1999

\begin{tabular}{|c|c|c|c|}
\hline Characteristic & & & \\
\hline \multirow[t]{2}{*}{ Gender $(N, \%)$} & Male & 8830 & 41.2 \\
\hline & Female & 12599 & 58.8 \\
\hline \multirow[t]{3}{*}{ Race $(N, \%)$} & White & 18188 & 84.9 \\
\hline & Black & 2301 & 10.7 \\
\hline & Other & 940 & 4.4 \\
\hline Low-income $(N, \%)$ & & 4637 & 21.6 \\
\hline \multirow[t]{2}{*}{ Age $(M, S D)$} & Male & 77.5 & 7.2 \\
\hline & Female & 80.0 & 7.9 \\
\hline Died during 1999 (N, \%) & & 7571 & 35.3 \\
\hline \# of hospitalizations $(M, S D)$ & & 2.96 & 2.20 \\
\hline \# of CHF hospitalizations $(M, S D)$ & & 1.38 & 0.86 \\
\hline Avg. Length of Stay $(M, S D)$ & & 7.3 & 6.9 \\
\hline Total Charges \$ US $(M, S D)$ & & 40,048 & 52,916 \\
\hline
\end{tabular}


Table 2. Estimates of Psychiatric Comorbidity For Patients With Heart Failure DRG At Any Hospitalization During $1999(\mathrm{~N}=21,429)$

\begin{tabular}{lccc}
\hline Type of Psychiatric Disorder & N & \% & $\begin{array}{c}\text { 95\% confidence } \\
\text { interval }\end{array}$ \\
\hline Any Psychiatric Disorder & 3287 & 15.34 & \\
Alcohol abuse & 290 & 1.4 & $1.2-1.5$ \\
Drug Abuse & 122 & 0.6 & $0.5-0.7$ \\
Psychosis & 534 & 2.5 & $2.3-2.7$ \\
Depression & 1821 & 8.5 & $0.2-0.3$ \\
Bipolar Disorders & 58 & 0.3 & $3.6-4.1$ \\
Anxiety Disorders & 816 & 3.8 & $1.0-1.3$ \\
Other Psychiatric Condition & 243 & 1.1 & \\
& & &
\end{tabular}


Table 3. Estimated Effects of Demographics, Medical and Psychiatric Comorbidities on Length of Stay, Number of Hospitalizations, and Total Hospitalization Charges For Patients With Heart Failure DRG (N = 21,429)

Estimated

Additional Number

(\% change)

of Hospitalizations
Estimated Additional

Mean Length of Stay

(\% change) in Days
Additional Total

Hospitalization Charges

Estimate

(US Dollars)

\begin{tabular}{|c|c|c|c|c|c|c|}
\hline Effect & $\begin{array}{l}\text { Parameter } \\
\text { Estimate }\end{array}$ & $\begin{array}{c}p \\
\text { value }\end{array}$ & $\begin{array}{l}\text { Parameter } \\
\text { Estimate }\end{array}$ & $\begin{array}{c}p \\
\text { value }\end{array}$ & Parameter Estimate & $\begin{array}{c}p \\
\text { value }\end{array}$ \\
\hline \multicolumn{7}{|l|}{ Demographics } \\
\hline Age $70-79 *$ & - & .408 & $0.39(+9 \%)$ & $<.0001$ & $-1001.29(-7 \%)$ & .006 \\
\hline Age $80-89 *$ & $0.09(+7 \%)$ & $<.0001$ & 1.37 (+31\%) & $<.0001$ & $-1662.65(-11 \%)$ & $<.0001$ \\
\hline Age 90+* & $0.15(+11 \%)$ & $<.0001$ & $2.30(+51 \%)$ & $<.0001$ & 2827.77 (+19\%) & $<.0001$ \\
\hline Female & $-0.03(-2 \%)$ & .016 & $0.39(+9 \%)$ & $<.0001$ & $-813.87(-5 \%)$ & .001 \\
\hline Black race** & $-0.09(-7 \%)$ & $<.0001$ & - & .713 & - & .159 \\
\hline Other race** & - & .317 & - & .101 & 3261.55 (+22\%) & $<.0001$ \\
\hline Medicaid & $0.08(+6 \%)$ & $<.0001$ & $0.37(+8 \%)$ & $<.0001$ & - & .682 \\
\hline \multicolumn{7}{|l|}{ Medical Comorbidities } \\
\hline Hypertension & $0.39(+30 \%)$ & $<.0001$ & $-0.30(-7 \%)$ & $<.0001$ & 5295.26 (+35\%) & $<.0001$ \\
\hline Valvular Disease & $0.21(+16 \%)$ & $<.0001$ & - & .455 & 4901.10 (+33\%) & $<.0001$ \\
\hline Pulmonary Circulation Disease & $0.17(+13 \%)$ & $<.0001$ & $0.38(+9 \%)$ & $<.0001$ & 2806.30 (+19\%) & $<.0001$ \\
\hline Peripheral Vascular Disease & $0.29(+23 \%)$ & $<.0001$ & $0.24(+5 \%)$ & .003 & 4320.65 (+29\%) & $<.0001$ \\
\hline Paralysis & $0.40(+31 \%)$ & $<.0001$ & $1.75(+39 \%)$ & $<.0001$ & $6613.03(+44 \%)$ & $<.0001$ \\
\hline
\end{tabular}


Other Neurological Disorders

Chronic Pulmonary Disease

Diabetes without Chronic

Complications

Diabetes with Chronic

Complications

Hypothyroidism

Renal Failure

Liver Disease

Lymphoma

Peptic Ulcer Disease and

Bleeding

Metastatic Cancer

Solid tumor without Metastasis

Rheumatoid arthritis/ Collagen

Vascular Diseases

Coagulation Deficiency

Obesity

Weight Loss

Fluid and electrolyte Disorders

Chronic Blood Loss Anemia

Deficiency Anemias

Acquired Immune Deficiency

Syndrome

\section{Psychiatric Comorbidities}

Alcohol abuse

$$
0.22(+17 \%) \quad<.0001
$$

$1.06(+24 \%) \quad<.0001$

$3987.54(+27 \%)$

$<.0001$

$0.30(+23 \%)<.0001$

$0.45(+10 \%) \quad<.0001$

$5548.66(+37 \%)$

$<.0001$

$$
0.16(+12 \%) \quad<.0001
$$$$
-\quad .08
$$

$1731.00(+12 \%)$

$<.0001$

$$
0.27(+21 \%) \quad<.0001
$$

$0.16(+13 \%)<.0001$

$0.22(+17 \%) \quad<.0001$

$0.23(+18 \%) \quad<.0001$

$0.15(+12 \%) \quad .004$
$0.81(+18 \%) \quad<.0001$

$-\quad .102$

$0.39(+9 \%)<.0001$

$0.53(+12 \%)$

— .772

.035

$2506.64(+17 \%)$

.019

$2854.62(+19 \%)$

.019

$\begin{array}{crrrcr}- & .141 & - & .777 & - & .723 \\ 0.29(+23 \%) & <.0001 & 0.65(+14 \%) & .002 & 3801.28(+25 \%) & <.0001\end{array}$

$0.31(+24 \%)<.0001$

- .311

$4355.15(+29 \%) \quad<.0001$

$$
0.27(+21 \%) \quad<.0001
$$

$0.71(+16 \%) \quad<.0001$

$3330.07(+22 \%)$

$<.0001$

$0.24(+19 \%) \quad<.0001$

$0.82(+18 \%) \quad<.0001$

$8465.06(+56 \%)$

$<.0001$

$0.15(+12 \%) \quad<.0001$

$0.41(+9 \%) \quad .002$

$1896.18(+13 \%)$

.001

$0.36(+28 \%)<.0001$

$1.91(+43 \%) \quad<.0001$

$11497.23(+76 \%)$

$<.0001$

$$
0.43(+33 \%) \quad<.0001
$$

$0.83(+18 \%) \quad<.0001$

$7381.35(+49 \%)$

$<.0001$

$0.31(+24 \%) \quad<.0001$

$0.33(+7 \%) \quad .021$

$4485.02(+30 \%)$

$<.0001$

$0.30(+24 \%)<.0001$

$0.22(+5 \%) \quad .001$

4202.99 (+28\%)

$<.0001$
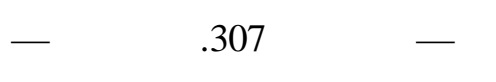

.744

.478

$0.21(+16 \%) \quad<.0001$

$0.58(+13 \%)$

.026

3461.25 (+23\%)

.002 
Drug Abuse

Psychoses

Depression

Anxiety Disorders

304

Other Psychiatric Condition
Bipolar Disorders
$0.32(+25 \%)<.0001$

$$
0.30(24 \%) \quad<.0001
$$

$0.39(+30 \%) \quad<.0001$

$0.38(+30 \%) \quad .001$

$0.34(+27 \%)<.0001$

$0.37(+29 \%)<.0001$
.819

$3723.24(+25 \%)$

.031

305

Notes:

306 Entries in Table 3 were calculated from GLM models and presented as actual hospitalizations, average LOS

307 in days, and total charges for ease of interpretation following the methods of Zhou, Stroupe and

308 Tierney $^{30}$. For parameters that were statistically significant, estimates represent the increased/decreased

309 utilization associated with the characteristic, in relation to the intercept value. In addition, the percent

310 increase in the estimate relative to the intercept value is also presented. The intercept values for the

311 outcomes in the table were as follows: 1.28 hospitalizations, 4.47 days of length of stay, and total charges of

$312 \$ 15,037.21$.

313

$314 *$ Age 65-69 is reference category.

$315 * *$ White race is reference category.

317 


\section{REFERENCES}

322

323 1. Rosamond W, Flegal K, Friday G, et al. Heart disease and stroke statistics--2007 update: a

324 report from the American Heart Association Statistics Committee and Stroke Statistics

325 Subcommittee. Circulation 2007;115(5):e69-171.

326 2. Silver MA. Advancing the advances in heart failure: updates on newer therapies. Congest

327 Heart Fail 2005;11(4):176.

328 3. Lee WC, Chavez YE, Baker T, et al. Economic burden of heart failure: a summary of

329 recent literature. Heart Lung 2004;33(6):362-371.

330 4. Garis RI, Farmer KC. Examining costs of chronic conditions in a Medicaid population.

331 Manag Care 2002;11(8):43-50.

332 5. Freedland KE, Rich MW, Skala JA, et al. Prevalence of Depression in Hospitalized

333 Patients With Congestive Heart Failure. Psychosom Med 2003;65(1):119-128.

334 6. Braunstein JB, Anderson GF, Gerstenblith G, et al. Noncardiac comorbidity increases

335 preventable hospitalizations and mortality among Medicare beneficiaries with chronic heart

336 failure.[see comment]. J Amer Coll Card 2003;42(7):1226-1233.

337 7. Haworth JE, Moniz-Cook E, Clark AL, et al. Prevalence and predictors of anxiety and

338 depression in a sample of chronic heart failure patients with left ventricular systolic dysfunction.

339 Eur J Heart Fail 2005;7(5):803-808.

340 8. Kessler RC, Chiu WT, Demler O, et al. Prevalence, Severity, and Comorbidity of 12-

341 Month DSM-IV Disorders in the National Comorbidity Survey Replication. Arch Gen Psychiatry 342 2005;62(6):617-627.

343 9. Borowsky SJ, Rubenstein LV, Meredith LS, et al. Who is at risk of nondetection of mental

344 health problems in primary care? J Gen Intern Med 2000;15(6):381-388.

345 10. Zuccala G, Marzetti E, Cesari M, et al. Correlates of cognitive impairment among patients 346 with heart failure: results of a multicenter survey. Amer J Med 2005;118(5):496-502.

347 11. Bennett SJ, Sauvé MJ. Cognitive deficits in patients with heart failure: a review of the 348 literature. J Cardiovasc Nurs 2003;18(3):219-242. 
349 12. Elixhauser A, Steiner C, Harris DR, et al. Comorbidity measures for use with 350 administrative data. Med Care 1998;36(1):8-27.

351 13. HCUP Comorbidity Software. Healthcare Cost and Utilization Project (HCUP). 2005

352 [accessed April 22, 2005]; Available from: http://www.hcup-

353 us.ahrq.gov/toolssoftware/comorbidity/comorbidity.jsp

354 14. Hanrahan N. What mental health services are used by older adults and who provides these 355 services? A descriptive study using 1999 Medicare claims data [Dissertation]. Boston: Boston 356 College; 2002.

357 15. Elixhauser A, Steiner C, Palmer L. Clinical Classifications Software (CCS). 2005 358 [accessed August 1, 2005]; Available from: http://www.hcup-us.ahrq.gov/toolssoftware/ccs/ccs.jsp 359 16. Health and Human Services. The 1999 HHS Poverty Guidelines. 1999 [accessed 360 September 20, 2000]; Available from: http://aspe.os.dhhs.gov/poverty/99poverty.htm

361 17. Satterthwaite FW. An Approximate Distribution of Estimates of Variance Components. 362 Biomet Bull 1946;2:110-114.

363 18. Manning W, Mullahy J. Estimating log moidels: To transform or not to transform? J Health 364 Econ 2001;20:461-494.

365 19. Moser DK, Worster PL. Effect of psychosocial factors on physiologic outcomes in patients 366 with heart failure. J Cardiovasc Nurs 2000;14(4):106-115.

367 20. Rich MW. Heart failure in the oldest patients: the impact of comorbid conditions. Amer J 368 Ger Card 2005;14(3):134-141.

369 21. Oslin DW, Datto CJ, Kallan MJ, et al. Association between medical comorbidity and 370 treatment outcomes in late-life depression. J Am Geriatr Soc 2002;50(5 (Print)):823-828.

371 22. Carney RM, Freedland KE, Eisen SA, et al. Major depression and medication adherence in 372 elderly patients with coronary artery disease. Health Psychol 1995;14(1):88-90.

373 23. Goldberg D, Privett M, Ustun B, et al. The effects of detection and treatment on the 374 outcome of major depression in primary care: a naturalistic study in 15 cities. The effects of 375 detection and treatment on the outcome of major depression in primary care: a naturalistic study in 37615 cities 1998;48(437):1840-1844.

377 24. Charney DS, Reynolds CF, 3rd, Lewis L, et al. Depression and Bipolar Support Alliance 378 consensus statement on the unmet needs in diagnosis and treatment of mood disorders in late life. 
379 Depression and Bipolar Support Alliance consensus statement on the unmet needs in diagnosis and 380 treatment of mood disorders in late life. 2003;60(7):664-672.

381 25. Slimmer LM, Lyness JM, Caine ED. Stress, medical illness, and depression. Semin Clin 382 Neuropsychiatry 2001;6(1):12-26.

383 26. Iezzoni LI, Foley SM, Daley J, et al. Comorbidities, complications, and coding bias. Does 384 the number of diagnosis codes matter in predicting in-hospital mortality?[see comment]. JAMA 385 1992;267(16):2197-2203.

386 27. Sohler NL, Bromet EJ. Does racial bias influence psychiatric diagnoses assigned at first 387 hospitalization? Does racial bias influence psychiatric diagnoses assigned at first hospitalization? 388 2003;38(8):463-472.

389 28. Koenig HG. Depression in hospitalized older patients with congestive heart failure. Gen 390 Hosp Psych 1998;20(1):29-43.

391 29. Sullivan M, Simon G, Spertus J, et al. Depression-related costs in heart failure care.[see 392 comment]. Arch Int Med 2002;162(16):1860-1866.

393 30. Zhou X-H, Stroupe KT, Tierney WM. Regression Analysis of Health Care Charges with 394 Heteroscedasticity. Appl Statistics 2001;50(3):303-312. 